\title{
Pleiotropic win-win mutations can rapidly evolve in a nascent cooperative community despite unfavorable conditions
}

\author{
Samuel F. M. Hart, Chi-Chun Chen, Wenying Shou" \\ Fred Hutchinson Cancer Research Center, Division of Basic Sciences, Seattle, WA 98109 \\ (\#: Author of correspondence, wenying.shou@gmail.com)
}

\begin{abstract}
Cooperation, paying a cost to benefit other individuals, is widespread. Cooperation can be promoted by pleiotropic "win-win" mutations which directly benefit self and partner. Previously, we showed that "partner-serving" should be defined as increased benefit supply rate per intake benefit (Hart \& Pineda et al., 2019). Here, we report that "win-win" mutations can rapidly evolve even in nascent cooperation under conditions unfavorable for cooperation. Specifically, in a well-mixed environment we evolved engineered yeast cooperative communities where two strains exchanged costly metabolites lysine and hypoxanthine. Among cells that consumed lysine and released hypoxanthine, ecm 21 mutations repeatedly arose. ecm 21 is "self-serving", improving self's growth rate in limiting lysine. ecm21 is also "partner-serving", increasing hypoxanthine release rate per lysine consumption and the steady state growth rate of partner. ecm 21 also arose in monocultures evolving in lysine-limited chemostats. Thus, even without any pressure to maintain cooperation, pleiotropic win-win mutations may readily evolve.
\end{abstract}

\section{Introduction}

Cooperation, paying a fitness cost to generate benefits available to others - is widespread and thought to drive major evolutionary transitions ${ }^{1,2}$. For example in multi-cellular organisms, different cells must cooperate with each other and refrain from dividing in a cancerous fashion to ensure the propagation of the germline ${ }^{3}$. Cooperation between species, or mutualistic cooperation, are also common ${ }^{4}$. In extreme cases, mutualistic cooperation are obligatory, i.e. 
cooperating partners depend on each other for survival 5,6. For example, insects and endosymbiotic bacteria exchange costly essential metabolites ${ }^{6,7}$.

Cooperation is vulnerable to "cheaters" who gain a fitness advantage over cooperators by consuming benefits without reciprocating fairly. Cancers are cheaters of multi-cellular organisms ${ }^{8}$, and rhizobia variants can cheat on their legume hosts ${ }^{9}$. How might cooperation survive cheaters?

Various mechanisms are known to protect cooperation against cheaters. In "partner choice", an individual preferentially interacts with cooperating partners over spatially-equivalent cheating partners ${ }^{2,10-12}$. For example, client fish observes cleaner fish cleaning other clients, and then chooses the cleaner fish that offers high-quality service (removing client parasites instead of client tissue) to interact with ${ }^{11}$.

For organisms lacking partner choice mechanisms, a spatially-structured environment can promote the origin and maintenance of cooperation ${ }^{2,13-17}$. This is because in a spatiallystructured environment neighbors repeatedly interact, and thus cheaters will eventually suffer as their neighbors perish ("partner fidelity feedback"). In a well-mixed environment, since all individuals share equal access to the cooperative benefit regardless of their contributions, cheaters are favored over cooperators ${ }^{15}$. An exception is that cooperators can stochastically purge cheaters if cooperators happen to be better adapted to an environmental stress than cheaters ${ }^{18-20}$. Finally, pleiotropy, where a single mutation affects multiple phenotypes, can stabilize cooperation if reducing benefit supply to partner also elicits a crippling effect on self ${ }^{21-}$ 24. For example, when the social amoeba Dictyostelium discoideum experience starvation, a fraction of the cells differentiate into a non-viable stalk in order to support the remaining cells to differentiation into viable spores. $\operatorname{dim} A$ mutants attempt to cheat by avoiding the stalk fate, but they also fail to form spores ${ }^{21}$. As another example, during quorum sensing in Pseudomonas aeruginosa, cooperators pay a fitness cost to secret proteases that break down extracellular proteins into usable amino acids. LasR mutants that "cheat" by not secreting protease also fail to metabolize adenosine for themselves ${ }^{22}$. In both cases, a gene, together with its associated network, links an individual's cooperation capacity to the individual's fitness, thus stabilizing cooperation. However, given the long evolutionary histories of these cooperative systems, it is unclear how easily such a genetic linkage can arise. 
Here, we investigate whether cooperation-stabilizing "win-win" mutations could arise in a nascent cooperative community growing in an environment unfavorable for cooperation. The

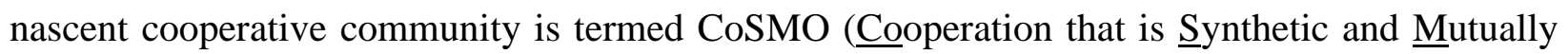
Obligatory). CoSMO comprises two non-mating engineered Saccharomyces cerevisiae strains: $L^{-}$ $H^{+}$requires lysine $(L)$ and pays a fitness cost to overproduce hypoxanthine $(H$, an adenine derivative) ${ }^{20,25}$, while $H^{-} L^{+}$requires hypoxanthine and pays a fitness cost to overproduce lysine 26 (Figure 1A). Overproduced metabolites are released into the environment by live cells ${ }^{25}$, allowing the two strains to feed each other. CoSMO models the metabolic cooperation between certain gut microbial species ${ }^{27}$ and between legumes and rhizobia ${ }^{28}$, as well as other mutualisms 29-34.

In our previous work, we allowed nine independent lines of CoSMO to evolve for over 100 generations in a well-mixed environment by performing periodic dilutions ${ }^{25,35}$. Throughout evolution, the two cooperating strains coexisted due to their metabolic co-dependence ${ }^{35,36}$. In a well-mixed environment, since partner-supplied benefits are uniformly distributed and equally available to all individuals, a self-serving mutation will be favored regardless of how it affects the partner. Indeed, all characterized mutants isolated from CoSMO displayed self-serving phenotypic changes ${ }^{20,25,26}$, outcompeting their ancestor in community-like environments. Here, we report the identification of a pleiotropic win-win mutation which is both self-serving and partner-serving. This win-win mutation also arose in the absence of the cooperative partner. Thus, cooperation-promoting win-win mutations can arise in a community without any evolutionary history of cooperation and in environments unfavorable to cooperation.

\section{Results}

\section{Criteria of a win-win mutation}

A win-win mutation is defined as a single mutation (e.g. a point mutation; a translocation; a chromosome duplication) that directly promotes the fitness of self ("self-serving") and the fitness of partner ("partner-serving"). To define "direct" here, we adapt the framework from Chapter 10 of (Peters et al., 2017): A mutation in genotype $A$ exerts a direct fitness effect on genotype $B$ if the mutation can alter the growth rate of $B$ even if the biomass of $A$ is fixed. 
For $\mathrm{L}^{-} \mathrm{H}^{+}$, a self-serving mutation should improve the growth rate of self by, for example, increasing cell's affinity for lysine (Figure 1B, orange). A self-serving mutation allows the mutant to outcompete a non-mutant. A partner-serving mutation should improve the growth rate of partner at a fixed self biomass. Since the partner requires hypoxanthine, a partner-serving mutation in $L^{-} H^{+}$should increase the hypoxanthine supply rate per $L^{-} H^{+}$biomass. Since the biomass of $\mathrm{L}^{-} \mathrm{H}^{+}$is linked to lysine consumption, the partner-serving phenotype of $L^{-} H^{+}$ translates to hypoxanthine supply rate per lysine consumption, or equivalently, hypoxanthine release rate per cell $\left(r_{H}\right)$ normalized by the amount of lysine consumed to make a cell $\left(c_{L}\right)^{26}$. We call this ratio " $H-L$ exchange ratio" (Figure $1 \mathrm{~B}$, purple). Note that a partner-serving mutation will eventually feedback to promote self growth. Indeed, after an initial lag, the growth rate of partner, of self, and of the entire community reach the same steady state growth rate $\sqrt{\frac{r_{H}}{c_{L}} \frac{r_{L}}{c_{H}}}$, where $r_{L}$ (lysine release rate per cell) and $c_{H}$ (hypoxanthine consumption per cell) are phenotypes of $H^{-} L^{+}$ 26.

\section{Community and monoculture evolution share similar mutations}

We randomly isolated evolved $\mathrm{L}^{-} \mathrm{H}^{+}$colonies from CoSMO, and subjected them to wholegenome sequencing. Nearly every sequenced clone harbored one or more of the following mutations: ecm21, rsp5, and duplication of chromosome 14 (DISOMY14) (Table 1, top), consistent with our earlier studies 20,25,26,37. Mutations in RSP5, an essential gene, mostly involved point mutations (e.g. $\operatorname{rsp} 5(P 772 L)$ ), while mutations in ECM21 mostly involved premature stop codons and frameshift mutations (Table 1, top; Table 1 Figure Supplement 1). Similar mutations also repeatedly arose when $\mathrm{L}^{-} \mathrm{H}^{+}$evolved as a monoculture in lysine-limited chemostats (Table 1, bottom), suggesting that these mutations emerged independently of the partner.

\section{Self-serving mutations increase the abundance of lysine permease}

Evolved $\mathrm{L}^{-} \mathrm{H}^{+}$clones are known to display a self-serving phenotype: they could form microcolonies on low-lysine plates where the ancestor failed to grow ${ }^{20,26}$. To quantify this selfserving phenotype, we used a fluorescence microscopy assay ${ }^{38}$ to measure the growth rates of ancestral and evolved $L^{-} H^{+}$in various concentrations of lysine. Under lysine limitation characteristic of the CoSMO environment (Figure 2A, "Comm. environ."), evolved $L^{-} H^{+}$clones 
containing an ecm 21 or $r s p 5$ mutation grew faster than a DISOMY14 strain which, as we showed previously, grew faster than the ancestor ${ }^{39}$. An engineered ecm $21 \Delta$ or $\operatorname{rsp} 5(P 772 L)$ mutation was sufficient to confer the self-serving phenotype (Figure 2A).

The self-serving phenotype is due to an increased abundance of the high-affinity lysine permease Lyp1 on the cell membrane. We have previously shown that duplication of the LYP1 gene, which resides on Chromosome 14, is necessary and sufficient for the self-serving phenotype of DISOMY14 (Figure 2A) ${ }^{39}$. Rsp5, an E3 ubiquitin ligase, is recruited by various "adaptor" proteins to ubiquitinate and target membrane transporters including Lyp1 for endocytosis and vacuolar degradation ${ }^{40}$. In high lysine, Lyp1-GFP was localized to cell membrane and vacuole in ancestral and ecm 21 cells, but localized to the cell membrane in rsp5 cells (Figure 2B, top row). Thus, Lyp1 localization was normal in ecm21 but not in rsp5, consistent with the notion that at high lysine concentration, Lyp1 is targeted for ubiquitination by Rsp5 through the Art1 instead of the Ecm 21 adaptor ${ }^{40}$. When ancestral $\mathrm{L}^{-} \mathrm{H}^{+}$was incubated in low lysine, Lyp1-GFP was initially localized on the cell membrane to facilitate lysine uptake, but later targeted to the vacuole for degradation and recycling ${ }^{41}$ (Figure 2B bottom panels). However, in both ecm 214 and $\operatorname{rsp5}(P 772 L)$ mutants, Lyp1-GFP was stabilized on cell membrane during prolonged lysine limitation (Figure 2B bottom panels), thus allowing mutants to grow faster than the ancestor during lysine limitation.

\section{ecm21 mutation is partner-serving}

The partner-serving phenotype of $\mathrm{L}^{-} \mathrm{H}^{+}$(i.e. hypoxanthine release rate per lysine consumption; exchange ratio $r_{H} / c_{L}$ ) can be measured in lysine-limited chemostats. In chemostats, fresh medium containing lysine was supplied at a fixed slow flow rate (mimicking the slow lysine supply by partner), and culture overflow exited the culture vessel at the same flow rate. After an initial lag, live and dead population densities reached a steady state (Figure 3- Figure supplement 1) and therefore, the net growth rate must be equal to the chemostat dilution rate dil (flow rate/culture volume). The released hypoxanthine also reached a steady state (Figure $3 \mathrm{~A}$ ). The $H$ - $L$ exchange ratio can be quantified as $d i l * H_{s S} / L_{0}{ }^{26}$, where $d i l$ is the chemostat dilution rate, $H_{s s}$ is the steady 
state hypoxantine concentration in the culture vessel, and $L_{0}$ is the lysine concentration in the inflow medium (which was fixed across all experiments).

Compared to the ancestor, ecm21 $1 \Delta$ but not DISOMY14 ${ }^{26}$ or $\operatorname{rsp} 5(P 772 L)$ exhibited increased $H$ $L$ exchange ratio. Specifically, at the same dilution rate (corresponding to 6-hr doubling), the steady state hypoxanthine concentration was the highest in ecm21s, and lower in the ancestor, DISOMY14 ${ }^{26}$, and $\operatorname{rsp5}(P 772 L)$ (Figure 3A). Although exchange ratio depends on doubling time, exchange ratios of ecm $21 \Delta$ consistently outperformed those of the ancestor across doubling times typically found in CoSMO (Figure 3B). Thus, compared to the ancestor, ecm $21 \Delta$ has a higher hypoxanthine release rate per lysine consumption.

To test whether ecm $21 \Delta$ can promote partner growth rate, we quantified the steady state growth

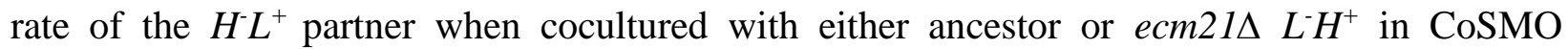
communities. After the initial lag, CoSMO reached a steady state growth rate ${ }^{39}$ (constant slopes in Figure 4A), which was also achieved by the two cooperating strains ${ }^{39}$. Compared to the ancestor, ecm $21 \Delta$ indeed sped up the steady state growth rate of CoSMO and of partner $\mathrm{HL}^{+}$ (Figure 4B). Thus, ecm $21 \Delta$ is partner-serving.

The partner-serving phenotype of ecm $21 \Delta$ can be explained by the increased hypoxanthine release rate per lysine consumption, rather than the evolution of any new metabolic interactions. Specifically, the growth rate of partner $\mathrm{HL}^{+}$(and of community) is approximately the geometric mean of the two strains' exchange ratios, or $\sqrt{\frac{r_{H}}{c_{L}} \frac{r_{L}}{c_{H}}}{ }^{25,39}$. Here, the ancestral partner's exchange ratio $\left(\frac{r_{L}}{c_{H}}\right)$ is fixed, while the exchange ratio of $L^{-} H^{+}\left(\frac{r_{H}}{c_{L}}\right)$ is $\sim 1.6$-fold increased in ecm $21 \Delta$ compared to the ancestor (at doubling times of $6 \sim 8 \mathrm{hrs}$; Figure 3B). Thus, ecm $21 \Delta$ is predicted to increase partner growth rate by $\sqrt{1.6}-1=26 \%$ (95\% confidence interval: $12 \% \sim 38 \%$; Figure 3 Source Data). In experiments, ecm $21 \Delta$ increased partner growth rate by $\sim 21 \%$ (Figure 4B; Figure 4 Source Data).

In conclusion, when $\mathrm{L}^{-} \mathrm{H}^{+}$evolved in nascent mutualistic communities and in chemostat monocultures in a well-mixed environment, win-win ecm 21 mutations repeatedly arose (Table 1). Thus, pleiotropic win-win mutations can emerge in the absence of any prior history of cooperation, and in environments unfavorable for cooperation. 


\section{Discussions}

Here, we demonstrate that pleiotropic win-win mutations can rapidly arise in conditions unfavorable for cooperation - such as in a well-mixed environment or in the absence of the cooperative partner. As expected, all evolved $\mathrm{L}^{-} \mathrm{H}^{+}$clones displayed self-serving phenotypes, achieving a higher growth rate than the ancestor in low lysine via stabilizing the lysine permease Lyp1 on cell membrane (Figure 2). Surprisingly, ecm21 mutants also displayed partner-serving phenotypes, promoting the steady state growth rate of partner $\mathrm{H}^{-} \mathrm{L}^{+}$and of community (Figure 4) via increasing the hypoxanthine release rate per lysine consumption (Figure 3).

The partner-serving phenotype of $\mathrm{L}^{-} \mathrm{H}^{+}$emerged as a side-effect of adaptation to lysine limitation instead of adaptation to a cooperative partner. We reached this conclusion because ecm 21 mutations were also observed in $\mathrm{L}^{-} \mathrm{H}^{+}$evolving as monocultures in lysine-limited chemostats (Table 1). Being self-serving does not automatically lead to a partner-serving phenotype. For example in the DISOMY14 mutant, duplication of the lysine permease LYP1 improved mutant's affinity for lysine (Figure 2) without improving hypoxanthine release rate per lysine consumption (Figure $3 \mathrm{~A}$ ) or partner's growth rate ${ }^{26}$.

How might ecm21 achieve higher hypoxanthine release rate per lysine consumption? One possibility is that purine overproduction is increased in ecm 21 mutants, leading to a steeper concentration gradient across the cell membrane. A different, and not mutually exclusive, possibility is that in ecm 21 mutants, purine permeases are stabilized much like the lysine permease, which in turn leads to an increased membrane permeability. Future work will reveal mechanisms of win-win mutations, as well as how common win-win mutations might be.

Here, we have defined a win-win mutation as a pleiotropic mutation that directly promotes the fitness of self and the fitness of partner. A broader definition of partner-serving is also possible if we include mutations that indirectly promote partner's fitness through promoting self fitness. Consider a mutant with improved affinity for lysine but no alterations in the metabolite exchange ratio (e.g. DISOMY14). By growing better in low lysine, this mutant will improve its own survival which in turn helps the whole community (and thus the partner) to survive the initial stage of low cell density. Indeed, all evolved $\mathrm{L}^{-} \mathrm{H}^{+}$clones tested so far improved community (and partner) viability in the sense that all mutants reduced the minimal total cell density required for the community to grow to saturation (Shou et al., 2007; Waite and Shou, 2012). Unlike ecm21, 
some of these mutations (e.g. DISOMY14) are not directly partner-serving, and would not improve partner's steady state growth rate ${ }^{26}$.

How likely might cooperation be stabilized by pleiotropy? Given that gene networks display "small world" connectivity ${ }^{42}$ and that a protein generally interacts with many other proteins, mutations in one gene will likely affect multiple cellular processes. Indeed, pleiotropy has been found to stabilize several natural cooperative systems ${ }^{21-24}$. In all these known examples, cooperation is intra-population, and has a long evolutionary history of cooperation. Our work demonstrates that pleiotropy can give rise to win-win mutations that promote nascent, mutualistic cooperation. The emergence of win-win mutations may not require elaborate evolutionary history, or selective pressure to maintain cooperation (e.g. in a well-mixed environment and even in the absence of the cooperative partner). In the absence of a cooperative partner, an abiotic environment could still sometimes mimic the community environment due to, for example, the presence of detritus. Adaptation to this abiotic environment may sometimes select for win-win mutations that will later benefit an incoming partner. Along with these earlier works, our study highlights the possibility of pleiotropy influencing the origin and maintenance of mutualistic cooperation. 


\section{Methods}

\section{Strains}

Our nomenclature of yeast genes, proteins, and mutations follows literature convention. For example, the wild type ECM21 gene encodes the Ecm21 protein; ecm21 represents a reductionof loss-of-function mutation. In our evolution experiments, we used $S$. cerevisiae strains of the RM11-1a background instead of the original S288C background ${ }^{35}$, because the latter had a significantly higher frequency of generating petites with defective mitochondria and compromised DNA-repair capability ${ }^{43}$. Both $\mathrm{L}^{-} \mathrm{H}^{+}$(WY1335) and $\mathrm{H}^{-} \mathrm{L}^{+}$(WY1340) were of MATa and harbored the ste $3 \Delta$ mutation (Table S1). Absence of the a-factor receptor Ste 3 would prevent mating between the two strains even after a rare event that switches a cell from MATa to MATalpha. We introduced desired genetic modifications into the ancestral RM11 background via transformation ${ }^{44,45}$. Strains were stored at $-80^{\circ} \mathrm{C}$ in $15 \%$ glycerol. All evolved or engineered strains used in this paper are summarized in Table 1 (evolved clones) and Supplementary File 1 (ancestral and engineered strains).

Growth medium and strain culturing have been previously discussed ${ }^{26}$.

\section{Experimental evolution}

CoSMO evolution has been described in detail in ${ }^{26}$. Briefly, exponentially-growing $L^{-} H^{+}$ (WY1335) and $H^{-} L^{+}$(WY1340) were washed free of supplements, counted using a Coulter counter, and mixed at 1000:1 (Line A), 1:1 (Line B), or 1:1000 (Line C) at a total density of $5 \times 10^{5} / \mathrm{ml}$. Three $3 \mathrm{ml}$ community replicates (replicates 1,2 , and 3 ) per initial ratio were initiated, thus constituting nine independent lines. Since the evolutionary outcomes of the nine lines were similar, they could be treated as a single group. Communities were grown at $30^{\circ} \mathrm{C}$ in glass tubes on a rotator to ensure well-mixing. Community turbidity was tracked by measuring the optical density $\left(\mathrm{OD}_{600}\right)$ in a spectrophotometer once to twice every day. In this study, $1 \mathrm{OD}$ was found to be $2 \sim 4 \times 10^{7}$ cells $/ \mathrm{ml}$. We diluted communities periodically to maintain OD at below 0.5 to avoid additional selections due to limitations of nutrients other than adenine or lysine. The fold-dilution was controlled to within 10 20 folds to minimize introducing severe population bottlenecks. Coculture generation was calculated from accumulative population density by multiplying OD 
with total fold-dilutions. Sample were periodically frozen down at $-80^{\circ} \mathrm{C}$. To isolate clones, a sample of frozen community was plated on rich medium YPD and clones from the two strains were distinguished by their fluorescence colors or drug resistance markers.

For chemostat evolution of $\mathrm{L}^{-} \mathrm{H}^{+}$, device fabrication and setup are described in detail in ${ }^{37}$. Briefly, the device allowed the evolution of six independent cultures, each at an independent doubling time. To inoculate each chemostat vessel, ancestral $L^{-} H^{+}$(WY1335) was grown to exponential phase in SD supplemented with $164 \mu \mathrm{M}$ lysine. The cultures were washed with SD and diluted to OD600 of $0.1\left(\sim 7 \times 10^{6} / \mathrm{ml}\right)$ in SD. $20 \mathrm{ml}$ of diluted culture was added to each vessel through the sampling needle, followed by $5 \mathrm{ml} \mathrm{SD}$ to rinse the needle of excess cells. Of six total chemostat vessels, each containing $\sim 43 \mathrm{~mL}$ running volume, three were set to operate at a target doubling time of 7 hours (flow rate $\sim 4.25 \mathrm{~mL} / \mathrm{hr}$ ), and three were set to an 11 hour target doubling time (flow rate $\sim 2.72 \mathrm{~mL} / \mathrm{hr}$ ). With $21 \mu \mathrm{M}$ lysine in the reservoir, the target steady state cell density was $7 \times 10^{6} / \mathrm{ml}$. In reality, live cell densities varied between $4 \times 10^{6} / \mathrm{ml}$ and $1.2 \times 10^{7} / \mathrm{ml}$. Samples were periodically taken through a sterile syringe needle. The nutrient reservoir was refilled when necessary by injecting media through a sterile 0.2 micron filter through a 60-ml syringe. We did not use any sterile filtered air, and were able to run the experiment without contamination for 500 hours. Some reservoirs (and thus vessels) became contaminated after 500 hours.

Whole-genome sequencing of evolved clones and data analysis were described in detail in ${ }^{26}$.

\section{Quantification methods}

Microscopy quantification of $\mathrm{L}^{-} \mathrm{H}^{+}$growth rates at various lysine concentrations was described in ${ }^{25,38}$. Briefly, cells were diluted into flat-bottom microtiter plates to low densities to minimize metabolite depletion during measurements. Microtiter plates were imaged periodically (every 0.5 2 hrs) under a 10x objective in a temperature-controlled Nikon Eclipse TE-2000U inverted fluorescence microscope. Time-lapse images were analyzed using an ImageJ plugin Bioact ${ }^{38}$. We normalized total fluorescence intensity against that at time zero, calculated the slope of $\ln$ (normalized total fluorescence intensity) over three to four consecutive time points, and chose the maximal value as the growth rate corresponding to the input lysine concentration. For validation of this method, see ${ }^{38}$. 
Short-term chemostat culturing of $\mathrm{L}^{-} \mathrm{H}^{+}$for measuring exchange ratio was described in ${ }^{25,38}$. Briefly, because $\mathrm{L}^{-} \mathrm{H}^{+}$rapidly evolved in lysine-limited chemostat, we took special care to ensure the rapid attainment of steady state so that an experiment is kept within $24 \mathrm{hrs}$. We set the pump flow rate to achieve the desired doubling time $T(19 \mathrm{ml}$ culture volume* $\ln (2) / T)$. Periodically, we sampled chemostats to measure live and dead cell densities, and the concentration of released hypoxanthine.

Cell density measurement via flow cytometry was described in ${ }^{25}$. Briefly, we mixed into each sample a fixed volume of fluorescent bead stock whose density was determined using a hemocytometer or Coulter counter. From the ratio between fluorescent cells or non-fluorescent cells to beads, we can calculate live cell density and dead cell density, respectively.

Chemical concentration measurement was performed via a yield-based bioassay ${ }^{25}$. Briefly, the hypoxanthine concentration in an unknown sample was inferred from a standard curve where the final turbidities of an ade- tester strain increased linearly with increasing concentrations of input hypoxanthine.

Quantification of CoSMO growth rate was described in ${ }^{25}$. Briefly, we used the "spot" setting where a $15 \mu \mathrm{l}$ drop of CoSMO community (1:1 strain ratio; $\sim 4 \times 10^{4}$ total cells/patch) was placed in a 4-mm inoculum radius in the center of a 1/6 Petri-dish agarose sector. During periodic sampling, we cut out the agarose patch containing cells, submerged it in water, vortexed for a few seconds, and discarded agarose. We then subjected the cell suspension to flow cytometry.

\section{Imaging of GFP localization}

Cells were grown to exponential phase in SD plus $164 \mu \mathrm{M}$ lysine. A sample was washed with and resuspended in SD. Cells were diluted into wells of a Nunc 96-well Optical Bottom Plate (Fisher Scientific, 165305) containing 300 $\mu \mathrm{l}$ SD supplemented with $164 \mu \mathrm{M}$ or $1 \mu \mathrm{M}$ lysine. Images were acquired under a $40 \mathrm{X}$ oil immersion objective in a Nikon Eclipse TE2000-U inverted fluorescence microscope equipped with a temperature-controlled chamber set at 300C. GFP was imaged using an ET-EYFP filter cube (Exciter: ET500/20x, Emitter: ET535/30m, Dichroic: T515LP). Identical exposure times (500 $\mathrm{msec})$ were used for both evolved and ancestral cells. 


\section{Introducing mutations into the essential gene RSP5}

Since RSP5 is an essential gene, the method of deleting the gene with a drug-resistance marker and then replacing the marker with a mutant gene cannot be applied. We therefore modified a two-step strategy ${ }^{46}$ to introduce a point mutation found in an evolved clone into the ancestral $\mathrm{L}^{-} \mathrm{H}^{+}$strain. First, a loxPkanMX-loxP drug resistance cassette was introduced into 300 bp after the stop codon of the mutant $r s p 5$ to avoid accidentally disrupting the remaining function in $r s p 5$. Second, a region spanning from $\sim 250 \mathrm{bp}$ upstream of the point mutation $[\mathrm{C}(2315) \rightarrow \mathrm{T}]$ to immediately after the loxP-kanMX-loxP drug resistance cassette was PCR-amplified. The PCR fragment was transformed into a wild-type strain lacking kanMX. G418-resistant colonies were selected and PCR verified for correct integration (11 out of 11 correct). The homologous region during transformation is large, and thus recombination can occur in such a way that the transformant got the KanMX marker but not the mutation. We therefore Sanger-sequenced the region, found that 1 out of 11 had the correct mutation, and proceeded with that strain. 


\section{Figures}

\section{Figure 1. Win-win mutation in a nascent cooperative community}

Figure 1 (A) CoSMO consists of two non-mating cross-feeding yeast strains, each engineered to overproduce a metabolite required by the partner strain. Metabolite overproduction is due to a mutation that renders the first enzyme of the biosynthetic pathway resistant to end-product inhibition ${ }^{47,48}$. Hypoxanthine and lysine are released by live $L^{-} H^{+}$and live $H^{-} L^{+}$cells at a rate of $r_{H}$ and $r_{L}$ per cell, respectively ${ }^{25}$, and are consumed by the partner at an amount of $c_{H}$ and $c_{L}$ per cell, respectively. The two strains can be distinguished by different fluorescent markers. (B) Win-win mutation. A win-win mutation is pleiotropic: it confers a self-serving phenotype (orange) and a partner-serving phenotype (lavender).

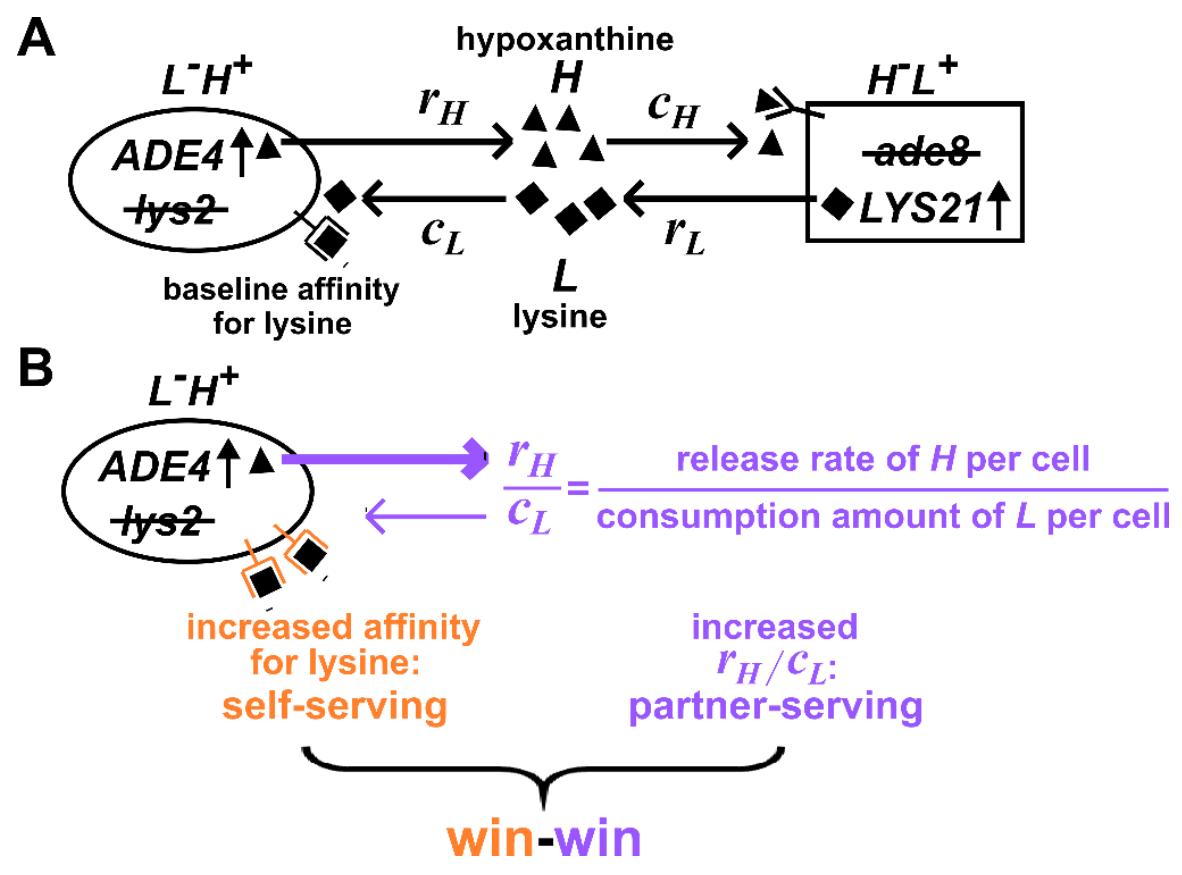




\section{Figure 2. Self-serving mutations increase growth rates at low lysine via increasing membrane Lyp1}

Figure 2 (A) Recurrent mutations are self-serving. We measured growth rates of mutant and ancestral strains in minimal SD medium with various lysine concentrations, using a calibrated fluorescence microscopy assay ${ }^{38}$. Briefly, for each sample, total fluorescence intensity of image frames were tracked over time, and the maximal positive slope of $\ln ($ fluorescence intensity) against time was used as the growth rate. Evolved strains grew faster than the ancestor in community environment (the grey dotted line demarcating "Comm. environ." corresponds to the lysine level supporting a growth rate of $0.1 / \mathrm{hr}$ as observed in ancestral CoSMO ${ }^{25}$ ). Measurements performed on independent days ( $\geq 3$ trials) were pooled and the average growth rate is plotted. Fit lines are based on Moser's equation for the birth rate $b$ as a function of metabolite concentration $L: b(L)=b_{\max } L^{n} /\left(K_{L}^{n}+L^{n}\right)$, where $b_{\max }$ is maximum birth rate in excess lysine, $K_{L}$ is the lysine concentration at which half maximum birth rate is achieved, and $n$ is the cooperitivity cooeficient describing the sigmoidal shape of the curve ${ }^{49}$. Evolved strains are marked with "evo"; engineered or backcrossed mutants are marked with the genotype. Data for DISOMY14 are reproduced from ${ }^{26}$ as a comparison. Data can be found in "Figure 2 Source Data". (B) Self-serving mutations stabilize Lyp1 localization on cell membrane. We fluorescently tagged Lyp1 with GFP in ancestor (WY1620), ecm214 (WY2355), and rsp5(P772L) (WY2356) to observe Lyp1 localization. We imaged each strain in a high lysine concentration $(164 \mu \mathrm{M})$ as well as after 4 and 10 hours incubation in low lysine $(1 \mu \mathrm{M})$. Note that low lysine was not consumed during incubation ${ }^{38}$. During prolonged lysine limitation, Lyp1 was stabilized to cell membrane in both mutants compared to the ancestor. 
bioRxiv preprint doi: https://doi.org/10.1101/2020.07.21.214130; this version posted July 21, 2020. The copyright holder for this preprint (which was not certified by peer review) is the author/funder, who has granted bioRxiv a license to display the preprint in perpetuity. It is made available under aCC-BY 4.0 International license.
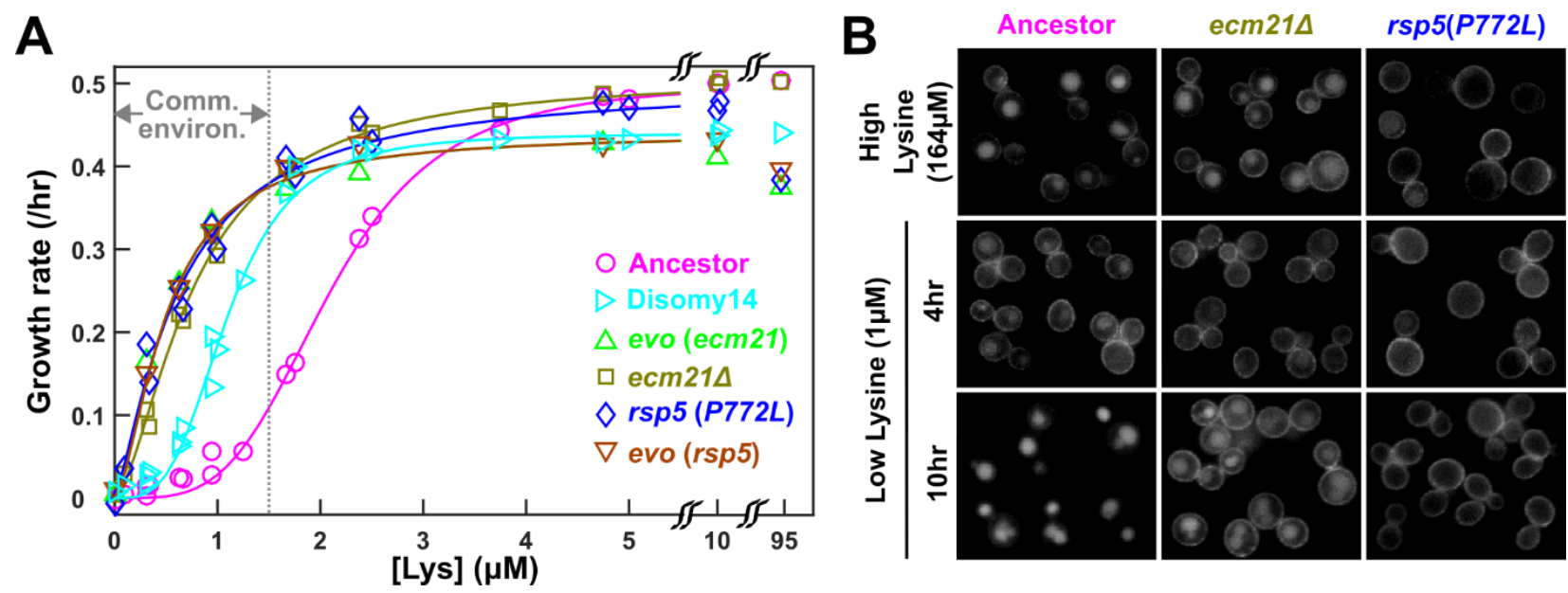


\section{Figure 3. ecm214 releases more hypoxanthine per lysine consumption.}

Figure 3 (A) Hypoxanthine accumulates to a higher level in ecm $21 \Delta$ chemostats than in ancestor chemostats. We cultured ancestor and mutant strains in lysine-limited chemostats (20 $\mu \mathrm{M}$ input lysine) at 6-hr doubling time (similar to CoSMO doubling time). Periodically, we quantified live and dead cell densities using flow cytometry (Figure 3 Figure Supplement 1), and hypoxanthine concentration in filtered supernatant using a yield-based bioassay ${ }^{25}$. The steady state hypoxanthine concentration created by the ancestor (WY1335) was lower than ecm21 (WY2226), and slightly higher than rsp5(P772L) (WY2475). DISOMY14 (WY2349) was indistinguishable from the ancestor, similar to our previous report ${ }^{26}$. (B) ecm $21 \Delta$ has a higher hypoxanthine-lysine exchange ratio than the ancestor. Cells were cultured in lysine-limited chemostats that spanned the range of CoSMO environments. In all tested doubling times, the exchange ratios of ecm $21 \Delta$ were significantly higher than those of the ancestor. The exchange ratios of $\operatorname{rsp5}(P 772 L)$ are similar to or lower than those of the ancestor. Mean and two standard deviations from 4 5 experiments are plotted. $p$-values are from two-tailed t-test assuming either unequal variance (4-hr doubling) or equal variance (6-hr and 8-hr doublings; verified by F-test). Data and $p$-value calculations can be found in "Figure 3 Source Data".

A

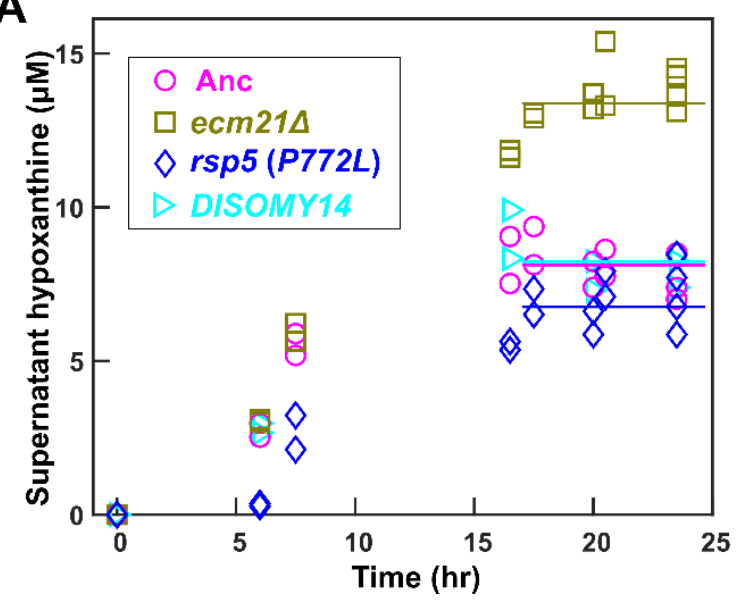

B

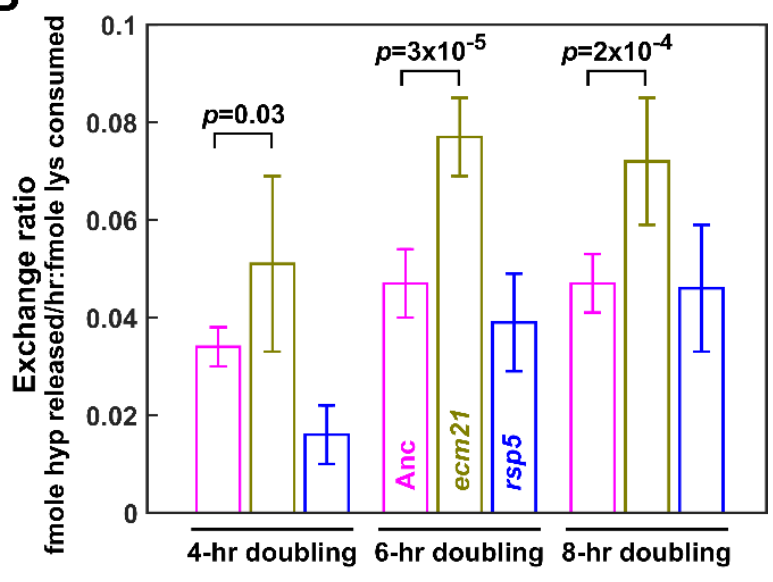


Figure 4. ecm 214 increases the growth rate of CoSMO and of partner.

To prevent rapid evolution, we grew CoSMO containing ancestral $H^{-} L^{+}$and ancestral or ecm21A mutant $\mathrm{L}^{-} \mathrm{H}^{+}$in a spatially-structured environment on agarose pads, and periodically measured the absolute abundance of the two strains using flow cytometry ${ }^{25}$. (A) Growth dynamics. After an initial lag, CoSMO achieved a steady state growth rate (slope of dotted line). (B) ecm21A increases the growth rate of CoSMO and of partner. Steady state growth rates of the entire community (left) and of partner $H^{-} L^{+}$(right) were measured $(\mathrm{n} \geq 6)$, and the average and two standard deviations are plotted. $p$-values are from two-tailed t-test with equal variance (verified by F-test). The full data set and outcomes of statistical tests can be found in Figure 4 Source Data.
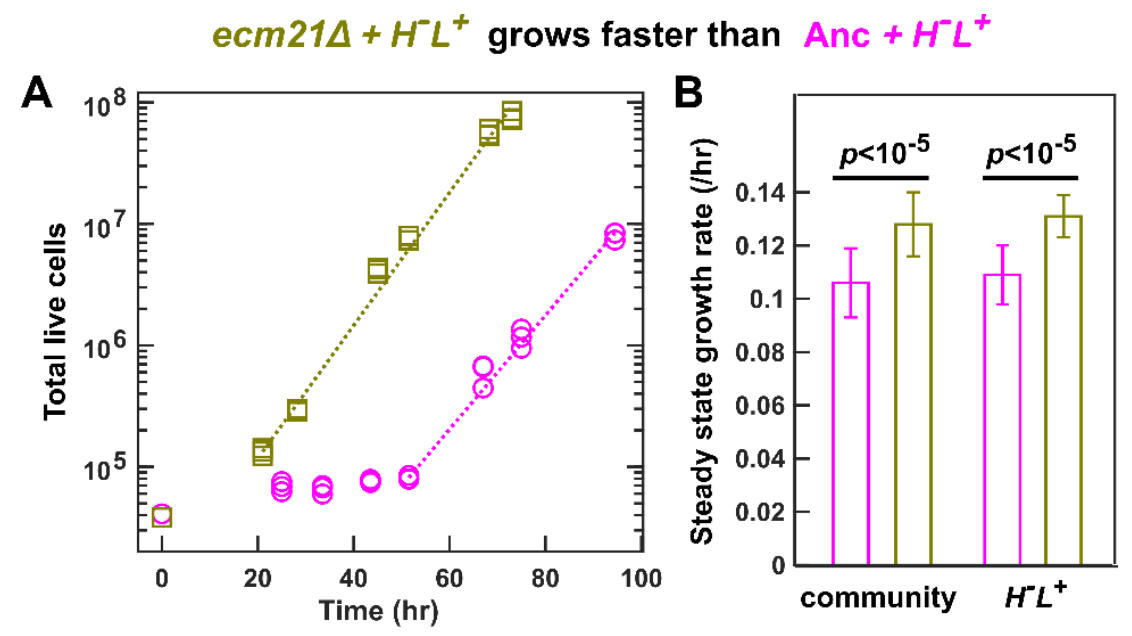


\section{Figure 3 Figure Supplement 1}

We cultured ancestor and mutant strains in lysine-limited chemostats ( $20 \mu \mathrm{M}$ input lysine) at 6hr doubling time (similar to CoSMO doubling time). Periodically, we measured live and dead cell densities using flow cytometry ${ }^{25}$. After a lag, live and dead cell densities reached a steady state. Data can be found in "Figure 3 Source Data".

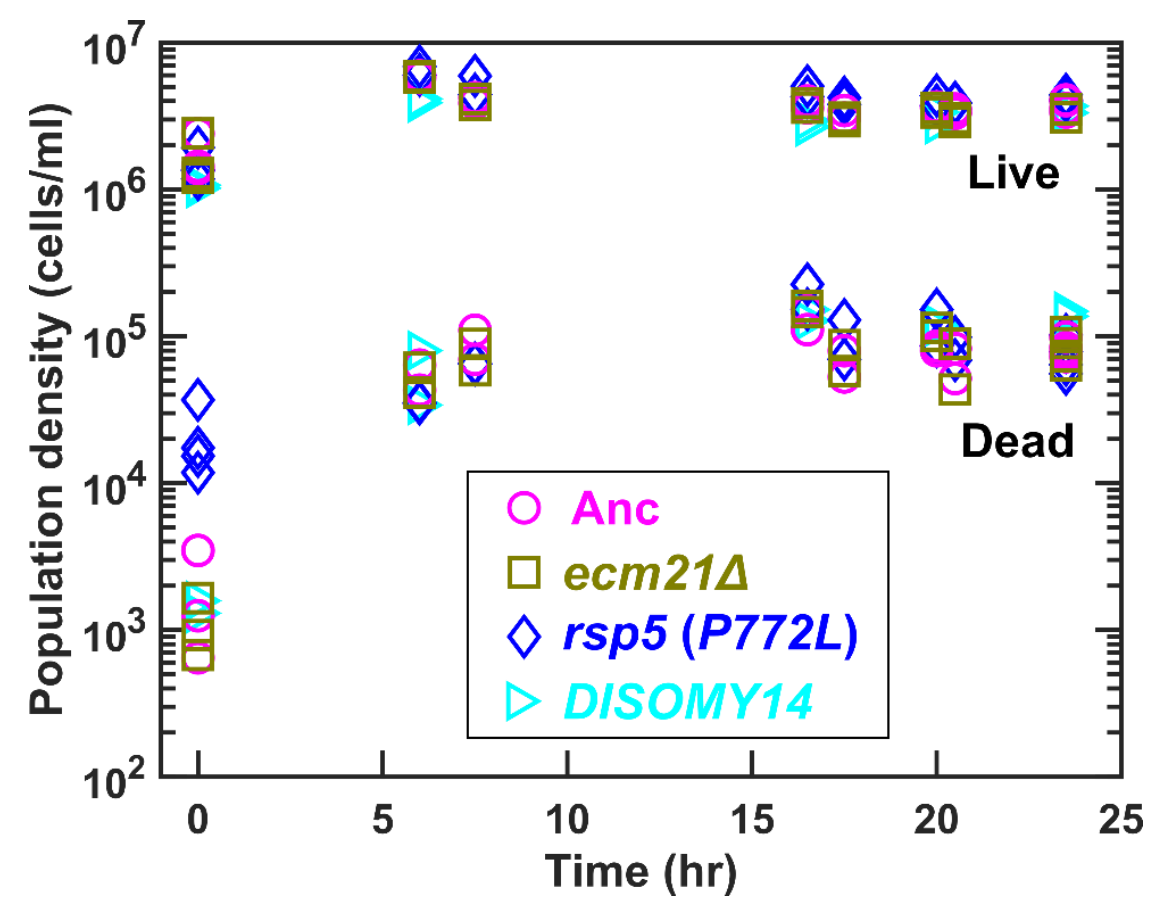




\section{Table 1. Mutations that repeatedly arose in independent lines}

Table 1 Single-nucleotide polymorphisms (SNPs) and chromosomal duplications from Illumina re-sequencing of $\mathrm{L}^{-} \mathrm{H}^{+}$from CoSMO communities (top) and lysine-limited chemostats (bottom). All clones except for two (WY1592 and WY1593 of line B3 at Generation 14) had either an ecm 21 or an rsp5 mutation, often in conjunction with chromosome 14 duplication. Note that the RM11 strain background in this study differed from the S288C strain background used in our earlier study ${ }^{20}$. This could explain, for example, why mutations in DOA4 were repeatedly observed in the earlier study ${ }^{20}$ but not here. For a schematic diagram of the locations of ecm 21 and rsp5 mutations with respect to protein functional domains, see Table 1-Figure Supplement 1. For other mutations, see Table 1-Table Supplement 1.

\begin{tabular}{|c|c|c|c|c|c|c|}
\hline$L-H_{+}$ & line & gen & ecm21 & rsp5 & $\begin{array}{c}\text { chromosome } \\
\text { duplicated }\end{array}$ & strain \\
\hline \multirow{14}{*}{$\begin{array}{l}\text { CoSMO } \\
\text { comm. }\end{array}$} & \multirow{4}{*}{ A1 } & \multirow{2}{*}{24} & Glu316 -> Stop & -- & 11,14 & WY1588 \\
\hline & & & -- & Pro772 -> Leu & 11 & WY1589 \\
\hline & & \multirow{2}{*}{151} & -- & Pro772 -> Leu & -- & WY1590 \\
\hline & & & -- & Pro772 -> Leu & $11,14,16$ & WY1591 \\
\hline & \multirow{5}{*}{ B1 } & 25 & Leu812->Stop & -- & 14 & WY1584 \\
\hline & & 49 & -- & Gly689 -> Cys & 14 & WY1585 \\
\hline & & \multirow{3}{*}{76} & -- & Gly689 -> Cys & -- & WY1586 \\
\hline & & & & Gly689 -> Cys & 14 & WY2467 \\
\hline & & & -- & Gly689 -> Cys & 14 & WY1587 \\
\hline & \multirow{5}{*}{ B3 } & \multirow{2}{*}{14} & -- & -- & -- & WY1592 \\
\hline & & & -- & -- & 14 & WY1593 \\
\hline & & \multirow{2}{*}{34} & Arg742 -> Stop & -- & 14,16 & WY1594 \\
\hline & & & Arg742 -> Stop & -- & 14,16 & WY1595 \\
\hline & & 63 & Arg742 -> Stop & Arg742 -> His & 12,14 & WY1596 \\
\hline \multirow{6}{*}{$\begin{array}{c}\text { lysine- } \\
\text { limited } \\
\text { chemostat } \\
\text { mono- } \\
\text { culture }\end{array}$} & \multirow{2}{*}{ 7.Line1 } & \multirow{2}{*}{30} & Asp652 frameshift & & 14 & WY1601 \\
\hline & & & Glu216 -> Stop & & 14 & WY1602 \\
\hline & 7.Line2 & 30 & Pro886 -> Ser & & $11,14,16$ & WY1603 \\
\hline & \multirow{2}{*}{ 7.Line3 } & \multirow{2}{*}{30} & Thr586 frameshift & & 14 & WY1604 \\
\hline & & & Thr586 frameshift & & 14 & WY1605 \\
\hline & 11.Line1 & 19 & Glu688 -> Stop & & 14,16 & WY1606 \\
\hline
\end{tabular}




\section{Table 1-Figure Supplement 1. Functional domains and positions of mutations in}

\section{Ecm 21 and Rsp5 proteins}

Mutations and their locations are marked with respect to the functional domains of the proteins. Numbers indicate amino acid positions, except in non-coding regions. Doman structures are obtained from the "protein" tab of SGD (https://www.yeastgenome.org/locus/S000000927/protein;

https://www.yeastgenome.org/locus/S000000197/protein). HECT domain is found in ubiquitin-protein ligases. WW domain can bind proteins with particular proline-motifs such as the PPxY motif. Arrestin Cterminal-like domain is involved in signaling and endocytosis of receptors. For ECM21, mutating the three poly-proline-tyrosine (PY) motifs after amino acid 884 inhibited the stress-induced endocytosis of the manganese transporter Smf1 ${ }^{50}$. Most ecm 21 mutations we recovered introduced premature stop codons before the PY motifs. In RSP5, the region including and upstream of - 470 is required for RSP5 function ${ }^{51}$. Mutations from coculture and monoculture isolates are marked above and below the gene, respectively.

\section{ECM21}

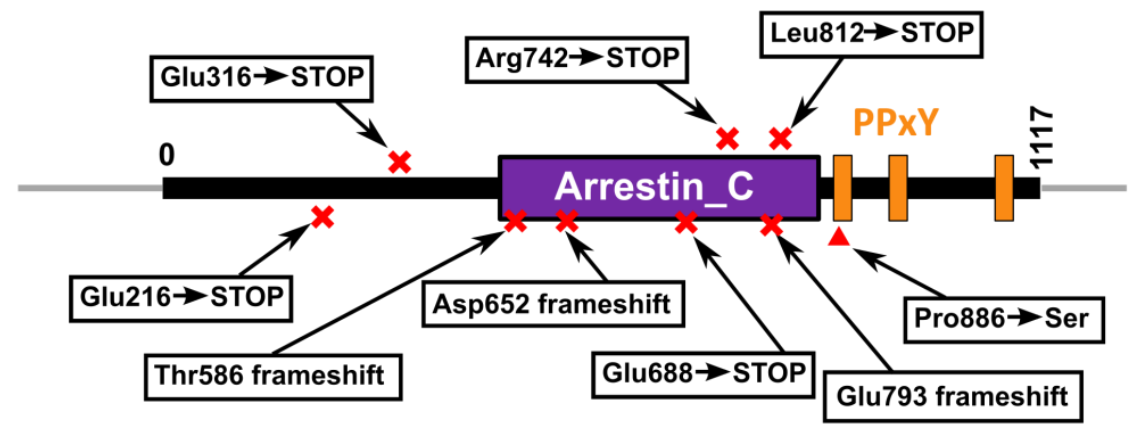

\section{RSP5}

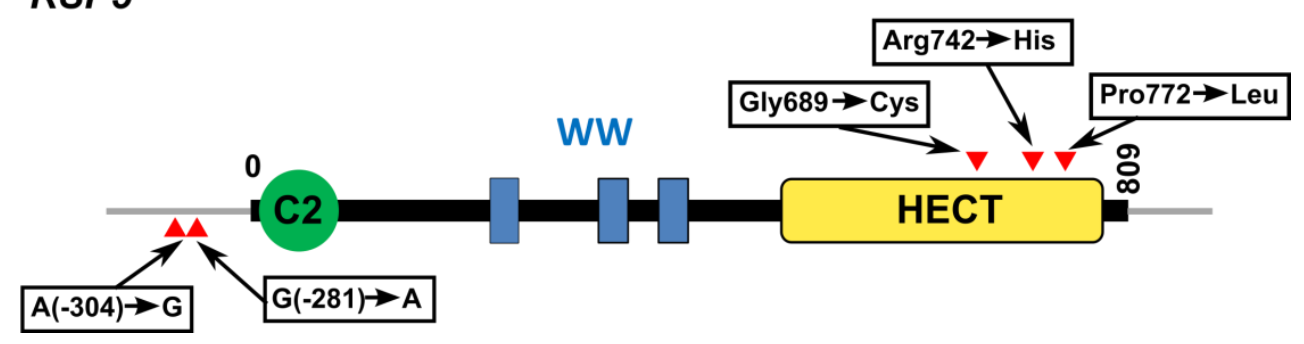




\title{
Table 1-Table Supplement 1. Summary of mutations
}

\author{
Supplementary file 1. List of strains
}

\section{Acknowledgement}

We thank Aric Capel for the chemostat evolution experiment data, and Jose Pineda for an earlier collaboration that eventually led to this discovery. We also thank members of the Shou lab (Li Xie, David Skelding, Alex Yuan, Sonal) for discussions.

\section{References}

1. Maynard Smith J. Evolution and the theory of games. Cambridge; New York: Cambridge University Press; 1982.

2. Sachs JL, Mueller UG, Wilcox TP, Bull JJ. The evolution of cooperation. The Quarterly Review of Biology. 2004;79(2):135-160.

3. Michod RE, Roze D. Cooperation and conflict in the evolution of multicellularity. Heredity. 2001 Jan;86(1):1-7.

4. Boucher DH. The Biology of Mutualism: Ecology and Evolution. Boucher DH, editor. New York: Oxford University Press; 1985.

5. Cook JM, Rasplus JY. Mutualists with attitude: coevolving fig wasps and figs (vol 18, pg 241, 2003). Trends in Ecology \& Evolution. 2003 Jul;18(7):325-325.

6. Zientz E, Dandekar T, Gross R. Metabolic interdependence of obligate intracellular bacteria and their insect hosts. Microbiology and Molecular Biology Reviews. 2004 Dec;68(4):745-770.

7. Gil R, Latorre A. Unity Makes Strength: A Review on Mutualistic Symbiosis in Representative Insect Clades. Life (Basel). 2019 Feb 25;9(1). PMID: 30823538

8. Aktipis C. Athena, Boddy Amy M., Jansen Gunther, Hibner Urszula, Hochberg Michael E., Maley Carlo C., Wilkinson Gerald S. Cancer across the tree of life: cooperation and cheating in multicellularity. Philosophical Transactions of the Royal Society B: Biological Sciences. $2015 \mathrm{Jul}$ 19;370(1673):20140219.

9. Gano-Cohen KA, Wendlandt CE, Stokes PJ, Blanton MA, Quides KW, Zomorrodian A, Adinata ES, Sachs JL. Interspecific conflict and the evolution of ineffective rhizobia. Ecol Lett. 2019 Mar 18; PMID: 30887662 
10. Kiers ET, Rousseau RA, West SA, Denison RF. Host sanctions and the legume-rhizobium mutualism. Nature. 2003 Sep 4;425(6953):78-81.

11. Bshary R, Grutter AS. Image scoring and cooperation in a cleaner fish mutualism. Nature. 2006 Jun 22;441(7096):975-978.

12. Shou W. Acknowledging selection at sub-organismal levels resolves controversy on procooperation mechanisms. eLife. 2015 Dec 29;4:e10106.

13. Chao L, Levin BR. Structured habitats and the evolution of anticompetitor toxins in bacteria. Proc Natl Acad Sci U S A. 1981 Oct;78(10):6324-8. PMCID: 349031

14. Momeni B, Waite AJ, Shou W. Spatial self-organization favors heterotypic cooperation over cheating. Elife. 2013;2:e00960. PMCID: PMC3823188

15. Harcombe W. Novel cooperation experimentally evolved between species. Evolution. 2010;64(7):2166-2172. PMID: 20100214

16. Nowak MA. Five Rules for the Evolution of Cooperation. Science. 2006 Dec 8;314(5805):1560-1563.

17. Pande S, Kaftan F, Lang S, Svatoš A, Germerodt S, Kost C. Privatization of cooperative benefits stabilizes mutualistic cross-feeding interactions in spatially structured environments. The ISME Journal. 2016 Jun;10(6):1413-1423.

18. Asfahl KL, Walsh J, Gilbert K, Schuster M. Non-social adaptation defers a tragedy of the commons in Pseudomonas aeruginosa quorum sensing. ISME J. 2015 Jan 23; PMID: 25615439

19. Morgan AD, Quigley BJZ, Brown SP, Buckling A. Selection on non-social traits limits the invasion of social cheats. Ecology Letters. 2012;15(8):841-846. PMID: 22639835

20. Waite AJ, Shou W. Adaptation to a new environment allows cooperators to purge cheaters stochastically. PNAS. 2012 Nov 20;109(47):19079-19086. PMID: 23091010

21. Foster KR, Shaulsky G, Strassmann JE, Queller DC, Thompson CR. Pleiotropy as a mechanism to stabilize cooperation. Nature. 2004 Oct 7;431(7009):693-6.

22. Dandekar AA, Chugani S, Greenberg EP. Bacterial quorum sensing and metabolic incentives to cooperate. Science. 2012 Oct 12;338(6104):264-266. PMID: 23066081

23. Oslizlo A, Stefanic $P$, Dogsa I, Mandic-Mulec I. Private link between signal and response in Bacillus subtilis quorum sensing. Proceedings of the National Academy of Sciences. 2014;111(4):15861591.

24. Harrison F, Buckling A. Siderophore production and biofilm formation as linked social traits. The ISME Journal. 2009 May;3(5):632-634.

25. Hart SFM, Mi H, Green R, Xie L, Pineda JMB, Momeni B, Shou W. Uncovering and resolving challenges of quantitative modeling in a simplified community of interacting cells. PLOS Biology. 2019 Feb 22;17(2):e3000135. 
26. Hart SFM, Pineda JMB, Chen C-C, Green R, Shou W. Disentangling strictly self-serving mutations from win-win mutations in a mutualistic microbial community. eLife. 2019 Jun 4;8:e44812.

27. Rakoff-Nahoum S, Foster KR, Comstock LE. The evolution of cooperation within the gut microbiota. Nature. 2016 Apr 25; PMID: 27111508

28. Schubert KR. Products of Biological Nitrogen Fixation in Higher Plants: Synthesis, Transport, and Metabolism. Annual Review of Plant Physiology. 1986;37(1):539-574.

29. Beliaev AS, Romine MF, Serres M, Bernstein HC, Linggi BE, Markillie LM, Isern NG, Chrisler WB, Kucek LA, Hill EA, Pinchuk GE, Bryant DA, Steven Wiley H, Fredrickson JK, Konopka A. Inference of interactions in cyanobacterial-heterotrophic co-cultures via transcriptome sequencing. ISME J. 2014 Nov;8(11):2243-2255.

30. Helliwell KE, Wheeler GL, Leptos KC, Goldstein RE, Smith AG. Insights into the Evolution of Vitamin B12 Auxotrophy from Sequenced Algal Genomes. Molecular Biology and Evolution. 2011 Oct 1;28(10):2921-2933.

31. Carini P, Campbell EO, Morré J, Sañudo-Wilhelmy SA, Cameron Thrash J, Bennett SE, Temperton B, Begley T, Giovannoni SJ. Discovery of a SAR11 growth requirement for thiamin's pyrimidine precursor and its distribution in the Sargasso Sea. ISME J. 2014 Aug;8(8):1727-1738.

32. Rodionova IA, Li X, Plymale AE, Motamedchaboki K, Konopka AE, Romine MF, Fredrickson JK, Osterman AL, Rodionov DA. Genomic distribution of B-vitamin auxotrophy and uptake transporters in environmental bacteria from the $\mathrm{C}$ hloroflexi phylum. Environmental microbiology reports. 2015;7(2):204-210.

33. Zengler K, Zaramela LS. The social network of microorganisms - how auxotrophies shape complex communities. Nature Reviews Microbiology. 2018 Mar 29;1.

34. Jiang $X$, Zerfaß C, Feng S, Eichmann R, Asally M, Schäfer P, Soyer OS. Impact of spatial organization on a novel auxotrophic interaction among soil microbes. The ISME Journal. 2018 Mar 23;1.

35. Shou W, Ram S, Vilar JM. Synthetic cooperation in engineered yeast populations. Proc Natl Acad Sci USA. 2007;104:1877-1882. PMCID: 1794266

36. Momeni B, Brileya KA, Fields MW, Shou W. Strong inter-population cooperation leads to partner intermixing in microbial communities. eLife. 2013;2:e00230. PMCID: 3552619

37. Green R, Wang L, Hart SF, Lu W, Skelding D, Burton JC, Mi H, Capel A, Chen HA, Lin A, Subramaniam AR, Rabinowitz JD, Shou W. Rapid evolution of an overt metabolic defect restores balanced growth. bioRxiv. 2018 Dec 17;498543.

38. Hart SFM, Skelding D, Waite AJ, Burton JC, Shou W. High-throughput quantification of microbial birth and death dynamics using fluorescence microscopy. Quant Biol [Internet]. 2019 Jan 4 [cited 2019 Feb 26]; Available from: https://doi.org/10.1007/s40484-018-0160-7

39. Hart SFM, Pineda JMB, Chen CC, Green R, Shou W. Disentangling strictly self-serving mutations from win-win mutations in a mutualistic microbial community. eLife, accepted. 2019; 
40. Lin CH, MacGurn JA, Chu T, Stefan CJ, Emr SD. Arrestin-Related Ubiquitin-Ligase Adaptors Regulate Endocytosis and Protein Turnover at the Cell Surface. Cell. 2008 Nov 14;135(4):714-725. PMID: 18976803

41. Jones CB, Ott EM, Keener JM, Curtiss M, Sandrin V, Babst M. Regulation of Membrane Protein Degradation by Starvation-Response Pathways. Traffic. 2012;13(3):468-482. PMID: 22118530

42. Boone $\mathrm{C}$, Bussey $\mathrm{H}$, Andrews BJ. Exploring genetic interactions and networks with yeast. Nat Rev Genet. 2007 Jun;8(6):437-449.

43. Veatch JR, McMurray MA, Nelson ZW, Gottschling DE. Mitochondrial dysfunction leads to nuclear genome instability via an iron-sulfur cluster defect. Cell. 2009 Jun 26;137(7):1247-1258. PMID: 19563757

44. Gietz RD, Woods RA. Transformation of yeast by lithium acetate/single-stranded carrier DNA/polyethylene glycol method. Meth Enzymol. 2002;350:87-96. PMID: 12073338

45. Waite AJ, Shou W. Constructing synthetic microbial communities to explore the ecology and evolution of symbiosis. Methods Mol Biol. 2014;1151:27-38. PMID: 24838876

46. Toulmay A, Schneiter R. A two-step method for the introduction of single or multiple defined point mutations into the genome of Saccharomyces cerevisiae. Yeast. 2006;23(11):825-831.

47. Armitt S, Woods RA. Purine-excreting mutants of Saccharomyces cerevisiae. I. Isolation and genetic analysis. Genet Res. 1970 Feb;15(1):7-17. PMID: 5415229

48. Feller A, Ramos F, Pierard A, Dubois E. In Saccharomyces cerevisae, feedback inhibition of homocitrate synthase isoenzymes by lysine modulates the activation of LYS gene expression by Lys14p. Eur J Biochem. 1999 Apr;261(1):163-70.

49. Skelding DH, Hart SFM, Vidyasagar T, Pozhitkov AE, Shou W. Developing a low-cost milliliter-scale chemostat array for precise control of cellular growth. Quant Biol. 2018 Jun 1;6:129-141.

50. Nikko E, Sullivan JA, Pelham HRB. Arrestin-like proteins mediate ubiquitination and endocytosis of the yeast metal transporter Smf1. EMBO reports. 2008 Dec 1;9(12):1216-1221.

51. Hein C, Springael JY, Volland C, Haguenauer-Tsapis R, André B. NPI1, an essential yeast gene involved in induced degradation of Gap1 and Fur4 permeases, encodes the Rsp5 ubiquitin-protein ligase. Mol Microbiol. 1995 Oct;18(1):77-87. PMID: 8596462 